ARTICLE

DOI: $10.1038 / \mathrm{s} 41467-018-02858-0$

OPEN

\title{
Cellular stressors contribute to the expansion of hematopoietic clones of varying leukemic potential
}

Terrence N. Wong et al.

Hematopoietic clones harboring specific mutations may expand over time. However, it remains unclear how different cellular stressors influence this expansion. Here we characterize clonal hematopoiesis after two different cellular stressors: cytotoxic therapy and hematopoietic transplantation. Cytotoxic therapy results in the expansion of clones carrying mutations in DNA damage response genes, including TP53 and PPM1D. Analyses of sorted populations show that these clones are typically multilineage and myeloid-biased. Following autologous transplantation, most clones persist with stable chimerism. However, DNMT3A mutant clones often expand, while PPM1D mutant clones often decrease in size. To assess the leukemic potential of these expanded clones, we genotyped $134 \mathrm{t}-\mathrm{AML} / \mathrm{t}-\mathrm{MDS}$ samples. Mutations in non-TP53 DNA damage response genes are infrequent in t-AML/t-MDS despite several being commonly identified after cytotoxic therapy. These data suggest that different hematopoietic stressors promote the expansion of distinct long-lived clones, carrying specific mutations, whose leukemic potential depends partially on the mutations they harbor.

Correspondence and requests for materials should be addressed to D.C.L. (email: dlink@dom.wustl.edu). ${ }^{*}$ A full list of authors and their affliations appears at the end of the paper. 
ematopoietic stem cells (HSCs) acquire somatic mutations with age, resulting in a genetically heterogeneous population with each HSC possessing its own set of unique mutations ${ }^{1}$. Some of these mutations confer a fitness advantage, allowing the HSCs harboring them to clonally expand. Indeed, expanded hematopoietic clones with specific mutations have been observed in healthy individuals without known hematologic abnormalities ${ }^{2-4}$. This has been termed clonal hematopoiesis of indeterminate potential ${ }^{5}$, and these clones have typically been found to carry mutations in epigenetic modifiers such as DNMT3A. Hematopoietic clonal expansion has also been observed following certain cellular stressors including aplastic anemia ${ }^{6}$ and cytotoxic therapy ${ }^{7,8}$. In these cases, the expanded hematopoietic clones often carried mutations in a different set of genes (i.e., PIGA, BCOR, and BCORL1 in the former and TP53 and $P P M 1 D$ in the latter), suggesting that cellular stressors influence hematopoietic clonal evolution. However, controlled studies investigating the degree to which specific stressors influence clonal expansion and characterizing the nature of these expanding hematopoietic clones are lacking.

To address these questions, we use a sensitive error-corrected sequencing approach to characterize clonal hematopoiesis after two distinct types of hematopoietic stress: cytotoxic therapy and hematopoietic autologous transplantation. We find that the stress from cytotoxic therapy promotes the expansion of clones with mutations in DNA damage response genes such as TP53 and $P P M 1 D$. Transplantation-related stress selects for a different set of hematopoietic clones, including those harboring DNMT3A mutations. Expanded clones are typically long-lived, multilineage, and transplantable. Finally, the leukemogenic potential of these clones is strongly dependent on the specific mutations they carry.

\section{Results}

Influence of cytotoxic therapy on clonal expansion. To assess how cytotoxic therapy influences the expansion of specific hematopoietic clones, we analyzed mobilized pre-transplant pheresis samples from three age and gender-matched cohorts: 69 lymphoma patients, 50 myeloma patients, and 19 healthy donors (Table 1, Supplementary Data 1 and Supplementary Table 1). Of the 119 patients with a history of malignancy, 81 (68 lymphoma patients and 13 myeloma patients) had previously received cytotoxic therapy (i.e., chemotherapy and/or radiation) and were grouped together. We sequenced 46 genes associated with clonal hematopoiesis or AML/MDS (Supplementary Table 2) using an error-corrected next-generation sequencing assay capable of detecting somatic mutations at a variant allele frequency (VAF) of $0.1 \%$. In total, 272 somatic nucleotide variants (SNVs) and 55 insertions/deletions (indels) were detected
(Supplementary Data 2). The median VAF of these variants was $0.5 \%$ (range: $0.1-22.6 \%$ ) and similar across genes (Supplementary Fig. 1A). Over eighty-six percent had a VAF $<2 \%$, the proposed threshold to detect clonal hematopoiesis ${ }^{5}$. Despite this lower VAF threshold, ninety percent of the identified SNVs were missense or nonsense mutations, and over $94 \%$ of the indels resulted in a frameshift (Supplementary Fig. 1B, 1C). This suggests that most of the identified variants had protein modifying activity.

Similar to earlier observations $s^{7,8}, 28.4 \%$ of patients exposed to cytotoxic therapy had clonal hematopoiesis carrying a mutation with a VAF $\geq 2 \%$; however, $82.7 \%$ of these same patients had clonal expansion with a VAF $\geq 0.1 \%$ (Fig. 1a). Both the incidence of clonal hematopoiesis and the number of somatic variants were higher in patients receiving cytotoxic therapy versus patients with malignancy not receiving cytotoxic therapy or healthy donors (Fig. 1a, b, Supplementary Table 3). This was not dependent on the patient's initial diagnosis, suggesting that the increase in identified variants was primarily due to the treatment they received (Supplementary Fig. 2). Consistent with prior reports, the number of variants correlated with age $\mathrm{a}^{2-4,7,8}$, regardless of antecedent cytotoxic therapy (Fig. 1c). Indeed, variants detected after cytotoxic therapy were predominantly transition mutations (Fig. 1d) and occurred in a similar trinucleotide context as variants identified in the absence of such exposure (Supplementary Fig. 1D), suggesting that they are age-related, not induced by cytotoxic drugs. As in studies with healthy individuals ${ }^{2-4}$, DNMT3A and TET2 variants were common in all cohorts (Fig. 1e). In contrast, consistent with studies on patients with a history of malignancy ${ }^{7,8}, T P 53$ and PPM1D variants were enriched after cytotoxic therapy (Supplementary Table 3) with all PPM1D variants being exon 6 nonsense or frameshift mutations (Supplementary Fig. 3).

Of the 46 genes interrogated, we identified six commonly associated with the DNA damage response pathway: TP53, PPM1D, ATM, BRCC3, SRCAP, and RAD21 ${ }^{9-12}$. Whereas 38/81 (46.9\%) of patients exposed to cytotoxic therapy had one or more variants in these genes, they were present in only 9/57 (15.8\%; $P=0.0001$ by Fisher's exact) of patients lacking such exposure (Fig. 1f). In contrast, variants in the other 40 genes were not significantly increased after cytotoxic therapy (Fig. 1g). Interestingly, 23/81 (28.4\%) of patients exposed to cytotoxic therapy had a variant in a DNA damage response gene other than TP53 or $P P M 1 D$. This was significantly higher than the $4 / 57$ (7.0\%; $P=$ 0.002 by Fisher's exact) of individuals without such exposure and suggests that exposure to cytotoxic therapy may confer a fitness advantage to hematopoietic clones harboring mutations in multiple genes involved in DNA damage response, not just clones with TP53 and PPM1D mutations.

In previous analyses of patients with hematologic and solid malignancies and concurrent clonal hematopoiesis, multiple

Table 1 Clinical summary of patients with error-corrected sequencing

\begin{tabular}{|c|c|c|c|c|}
\hline Age & Median (range) (years) & $\begin{array}{l}\text { Lymphoma }(n=69) \\
57(18-72)\end{array}$ & $\begin{array}{l}\text { Myeloma }(n=50) \\
63(26-76)\end{array}$ & $\begin{array}{l}\text { Healthy donor }(n=19) \\
60(21-76)\end{array}$ \\
\hline Gender & Male & $60.9 \%$ & $60.0 \%$ & $63.2 \%$ \\
\hline \multirow[t]{5}{*}{ Known previous treatment } & Cytotoxic chemotherapy & $98.6 \%$ & $4.0 \%$ & N.A. \\
\hline & Alkylator & $95.7 \%$ & $0.0 \%$ & N.A. \\
\hline & Topoisomerase II inhibitor & $95.7 \%$ & $4.0 \%$ & N.A. \\
\hline & Platinum & $59.4 \%$ & $0.0 \%$ & N.A. \\
\hline & Lenalidamide/thalidomide & $0.0 \%$ & $84.0 \%$ & N.A. \\
\hline Time from start of cytotoxic therapy to transplant & Median (range) (months) & $11(3-288)$ & N.A. & N.A. \\
\hline
\end{tabular}


a

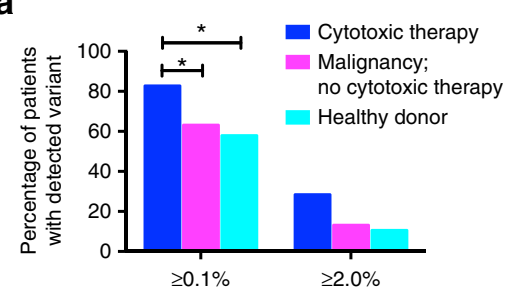

C

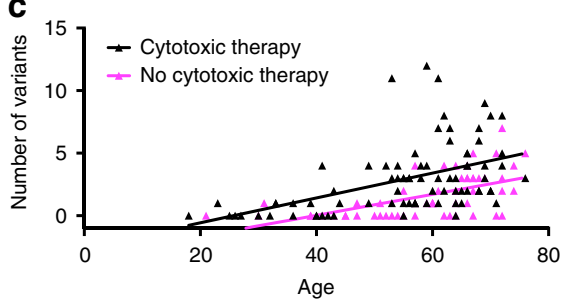

e

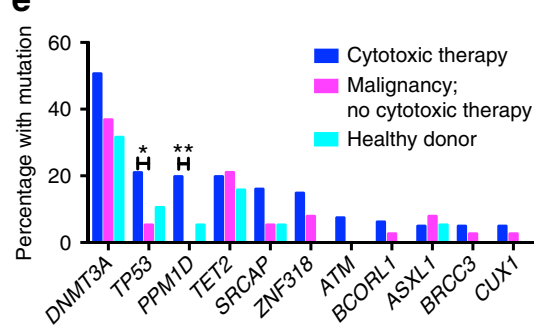

g

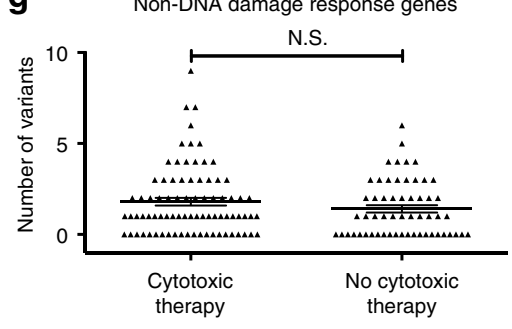

b

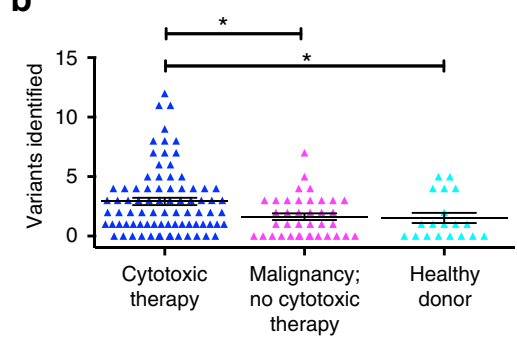

d

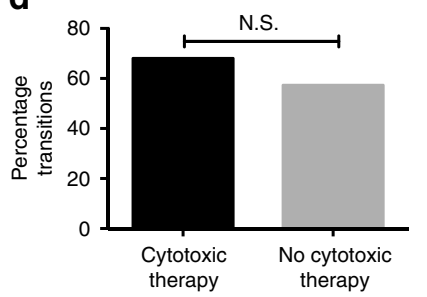

f

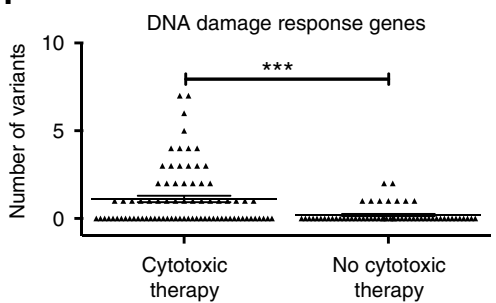

h

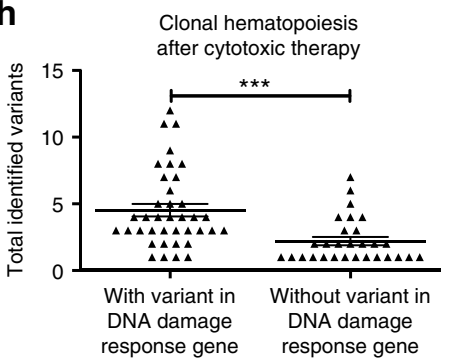

Fig. 1 Influence of cytotoxic therapy on clonal expansion. a Percentage of individuals with clonal hematopoiesis $\geq 0.1 \%$ or $\geq 2.0 \%$ in three cohorts: those exposed to cytotoxic therapy $(n=81)$, those with malignancy but not exposed to cytotoxic therapy $(n=38)$, and healthy donors $(n=19)$. The average ages for the three groups were 55.0, 60.1, and 55.8 years, respectively. b Number of detected variants per patient. c Number of identified variants plotted against individual age for all pheresis samples. $\mathbf{d}$ Percentage of variants detected in pheresis samples that were transition mutations. e Percentage of individuals with at least one expanded clone harboring a variant in the specified gene. $\mathbf{f}$ Total number of variants identified in DNA damage response genes (i.e., TP53, PPM1D, ATM, BRCC3, SRCAP, or RAD21) grouped by whether or not the individual had previously been exposed to cytotoxic therapy. $\mathbf{g}$ Same as in $\mathbf{f}$ except for all other variants (i.e., in "non-DNA damage response genes"). $\mathbf{h}$ Total number of variants detected in patients with clonal hematopoiesis following cytotoxic therapy grouped by whether or not at least one variant was in a DNA damage response gene. NS not significant. ${ }^{\star} P<0.05$; ${ }^{\star \star} P<0.01$; ${ }^{\star \star \star} P<0.001$. For $\mathbf{a}, \mathbf{d}, \mathbf{e}$, significance was determined with a Fisher's exact test. For $\mathbf{b}$, significance was determined with a negative binomial regression analysis using Bonferroni-adjusted pairwise comparisons. For $\mathbf{f}-\mathbf{h}$, significance was determined with a negative-binomial regression analysis. Data is represented as the mean \pm the standard error of the mean

variants were identified in 30.8 and $38 \%$ of patients, respectively ${ }^{7,8}$. With the increased sensitivity of our errorcorrected sequencing approach, we identified multiple variants in $73.1 \%(49 / 67)$ of patients with clonal hematopoiesis after cytotoxic therapy (range: $2-12$ variants), including 21 patients (31.3\%) with multiple variants in DNA damage response genes (Fig. 1b, Supplementary Fig. 4). Of those individuals with multiple variants, $59.2 \%(29 / 49)$ had multiple variants in the same gene, with 13 patients (26.5\%) having three or more (maximum: seven variants). As previously demonstrated with PPM1D alone ${ }^{8}$, patients with at least one mutation in a DNA damage response gene after cytotoxic therapy had a higher number of total variants than those with clonal hematopoiesis due to other mutations (Fig. 1h).

Expanded clones are usually multi-lineage and myeloid-biased. To investigate the nature of expanded mutant clones, we assessed the hematopoietic populations carrying their mutations. In preleukemic clones, the founding mutation is often in both myeloid and $\mathrm{T}$ cell compartments, suggesting that they arise in progenitors with multilineage potential ${ }^{13-15}$. We performed similar analyses with lymphoma patients after cytotoxic therapy. Myeloid and lymphoid populations were sorted from pheresis samples 
with DNMT3A, PPM1D, or TP53 mutations, and droplet digital PCR (ddPCR) was performed to quantify VAFs (Supplementary Fig. 5 and 6). Of note, B cells were often undetectable, reflecting recent rituximab exposure. As previously reported ${ }^{16}, D N M T 3 A$ mutations were detected in both myeloid and lymphoid cells, suggesting they arose in HSCs (Fig. 2a). With one exception (UPN-746), PPM1D and TP53 mutations were also detected in both myeloid and lymphoid cells, suggesting that they likewise arose in HSCs (Fig. 2b, c). Interestingly, the percentage of cells carrying the TP53 or PPM1D variant was higher (median: 14.2fold; range: 2.3 to 88.3 -fold; $P=0.03$ by Wilcoxon match-paired signed rank test) in myeloid versus $T$ cells. This suggests that the TP53 and PPM1D variants carried by hematopoietic clones expanding after cytotoxic therapy are initially and rapidly propagated through the myeloid lineages. Either the progenitors harboring these variants were myeloid-biased or didn't have time to fully populate the lymphoid lineages, particularly $\mathrm{T}$ cells, which are slower to turn over. More than half of our samples were collected within 1 year of cytotoxic therapy initiation, including UPN-746 (Fig. 2, Supplementary Data 1).

Influence of transplantation on clonal expansion. We then asked how transplantation influences the expansion of these HSCs. Transplantation causes replicative stress ${ }^{17,18}$ and alters the bone marrow microenvironment ${ }^{19-21}$, potentially influencing the competitive fitness of HSCs. Forty lymphoma patients (with 120 detected pheresis variants among 31 individuals) had peripheral blood samples collected 6-12 months after autologous transplantation. Most of these variants did not change significantly in VAF upon transplantation with their post-transplant VAFs strongly correlated to their VAFs before transplantation $\left(R^{2}=\right.$ 0.64 by linear regression); however, variants not initially identified in the pheresis samples often became detectable posttransplant (Fig. 3a, b, Supplementary Data 3). In total, 148 variants were called in 35 patients following autologous transplantation with the average number of variants per patient increasing slightly from $3.0 \pm 3.1$ to $3.7 \pm 3.2(P<0.05$ by Wilcoxon matchpaired signed rank test).

The two genes with the most variants identified either before or after transplant were DNMT3A and PPM1D. Of 51 DNMT3A variants, 17 (33.3\%) significantly increased $\geq 2$-fold in VAF after transplantation, while $3(5.9 \%)$ decreased (Fig. 3c, e). Interestingly, the VAFs of all three R882 codon DNMT3A variants increased. This suggests that certain DNMT3A variants confer a modest repopulating advantage with transplantation. In contrast, of 23 PPM1D variants, only $2(8.7 \%)$ significantly increased in VAF with transplantation, while 7 (30.4\%) decreased (Fig. 3d, e). Collectively, these data indicate that hematopoietic clones expanding after cytotoxic therapy are often long-lived, persisting following transplant. At the same time, previously unidentified clones may expand under the stress of transplantation. The behavior of hematopoietic clones following transplantation depends, in part, on what mutations they harbor; transplantation-induced selection pressure doesn't necessarily favor the same clones as cytotoxic therapy.
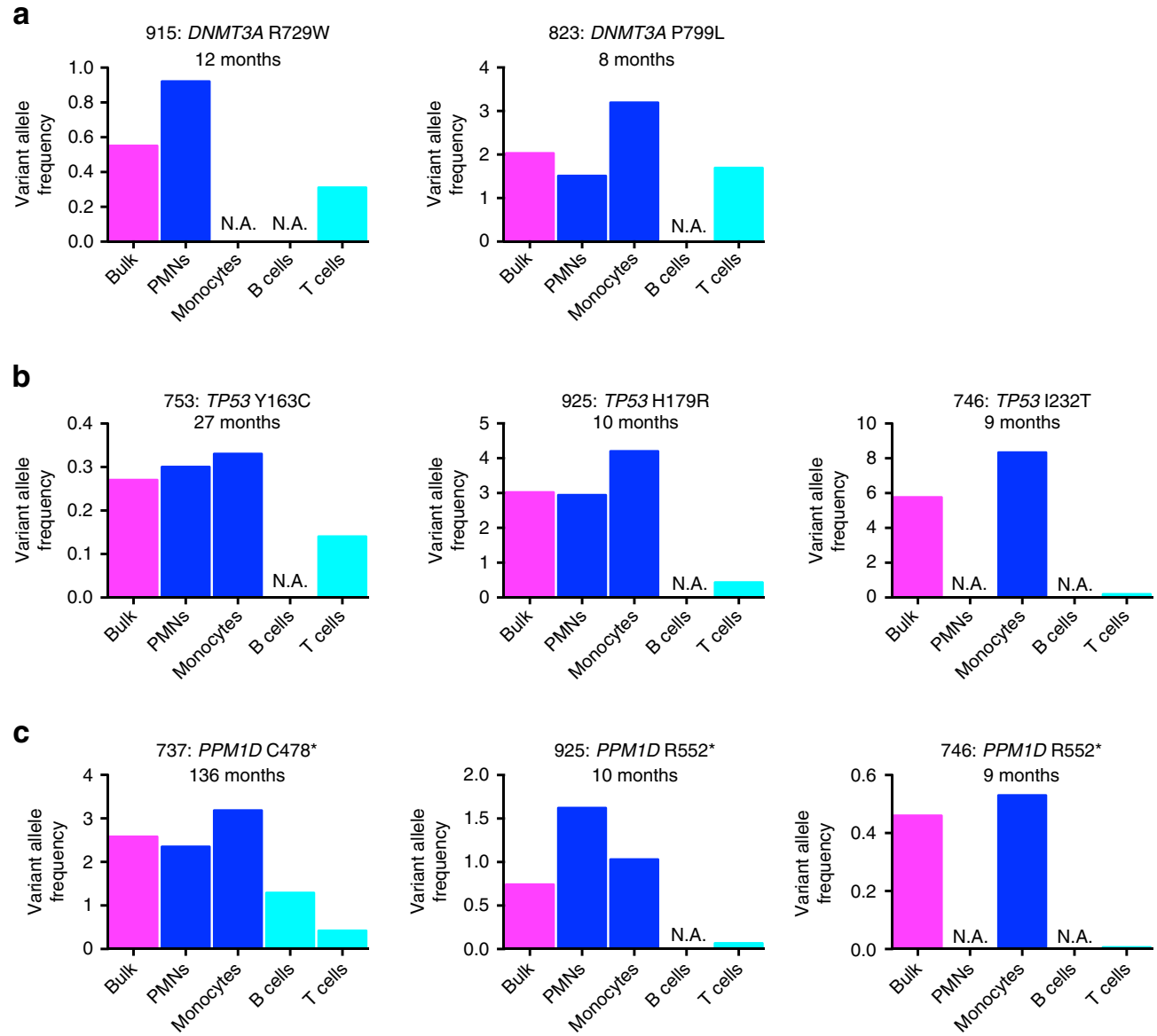

Fig. 2 Expanded clones are usually multi-lineage and myeloid-biased. VAFs of the specified DNMT3A (a), TP53 (b) or PPM1D (c) mutations in bulk cells (magenta) or in sorted neutrophils (PMNs, blue), monocytes (blue), B cells (turquoise), or T cells (turquoise). N.A.: the hematopoietic population was not able to be obtained. The latency from the time of first cytotoxic therapy exposure to autologous stem cell transplant is noted 
a

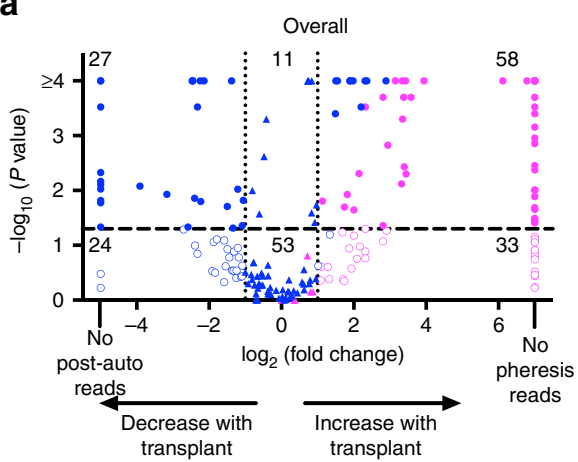

C

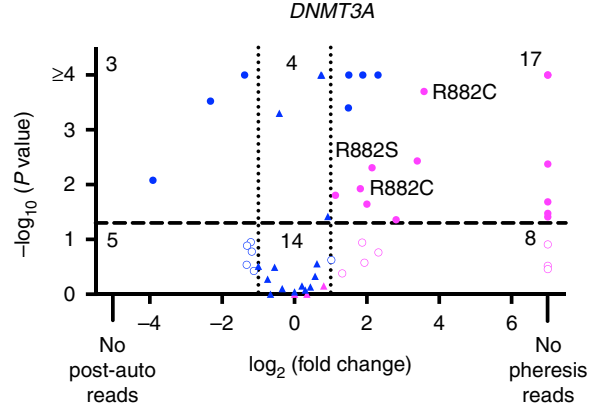

b

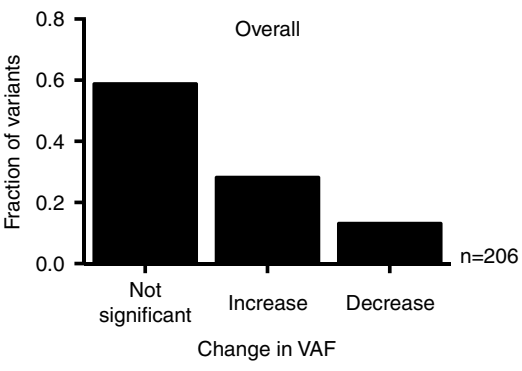

d

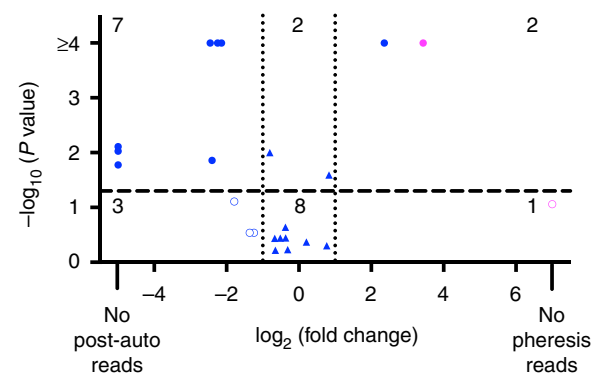

e

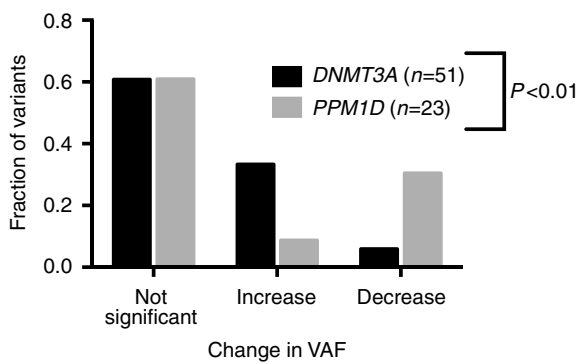

Fig. 3 Influence of transplantation on clonal expansion. a Volcano plot showing the fold change in VAF following transplantation for those variants initially identified in pre-transplant pheresis samples (in blue) and those only detected after transplant (in magenta). Dotted and dashed lines respectively represent a greater than two-fold change in VAF and $P<0.05$ by Fisher's exact or $\chi^{2}$ tests. Triangles indicate a less than two-fold change in VAF, while circles indicate a greater than two-fold change. Variants with open circles had a greater than two-fold change but with $P>0.05$. $\mathbf{b}$, Summary of the changes in VAF after transplantation for all genes; changes in VAF were deemed significant if they were $>2$-fold with a $P$ value $<0.05$. c, $\mathbf{d}$ Same as a except focusing on DNMT3A (c) or PPM1D (d) variants. e Same as b except summarizing the changes in VAF for DNMT3A and PPM1D variants. Significance for the difference in behavior after transplantation between variants in DNMT3A and PPM1D was determined with a $\chi^{2}$ analysis

Leukemogenic potential of expanded clones. Clonal hematopoietic expansion has been linked to the development of hematologic malignancies both with and without previous cytotoxic therapy exposure $2,3,8,22,23$. However, it remains unclear which clones are at highest risk of leukemic transformation. At a median follow-up of $\sim 2.2$ years, no patient in our lymphoma cohort had developed

t-AML/t-MDS, so directly assessing the leukemogenic potential of expanded clones following cytotoxic therapy was not possible. Instead, we indirectly assessed this potential by determining how frequently these genes are mutated in $t$-AML/t-MDS. We performed exome sequencing on 19 cases and targeted sequencing on 92 cases with a panel of 274 genes, including the DNA damageassociated genes assessed in our error-corrected HaloPlex assay (Supplementary Table 4). Combined with our previously published t-AML/t-MDS whole genome and exome sequencing 24 , we analyzed 134 cases in total (Table 2, Supplementary Data 4).

As in previous reports 24,25 , these patients had poor overall survival (Supplementary Fig. 7). They frequently harbored mutations in epigenetic modifiers, spliceosome genes, and myeloid transcription factors and were enriched in TP53 mutations (Fig. 4a, Supplementary Data 5). The null hypothesis that all gene mutations confer a similar risk of progression from clonal hematopoiesis to $t$-AML/t-MDS predicts that the frequency of gene mutations in therapy-related clonal hematopoiesis will be similar to that in $t-A M L / t-M D S$. However, this pattern was not observed as illustrated by DNA damage response genes (Fig. 4b). TP53 mutations were frequently observed in both therapy-related clonal hematopoiesis and in t-AML/t-MDS. In contrast, mutations in PPM1D and SRCAP, which were present at approximately the same frequency as TP53 mutations in therapyrelated clonal hematopoiesis, were uncommon in t-AML/t-MDS. Similar trends were observed upon restricting t-AML/t-MDS patients to those with a previous history of lymphoma (Fig. 4c). This suggests that the leukemogenic potential of expanded clones carrying different mutations varies significantly. Specifically, the leukemogenic potential of expanded TP53 mutant hematopoietic 


\section{Table 2 Clinical summary of t-AML/t-MDS patients}

\begin{tabular}{|c|c|c|c|c|}
\hline \multirow[b]{2}{*}{ Age } & $\begin{array}{l}\text { T-AML/t-MDS } \\
\text { sequencing } \\
\text { cohort }\end{array}$ & $\begin{array}{l}\text { Overall } \\
(n=134)\end{array}$ & $\begin{array}{l}\text { Exome } \\
(n=20)\end{array}$ & $\begin{array}{l}\text { Targeted } \\
(n=92)\end{array}$ \\
\hline & $\begin{array}{l}\text { Median } \\
\text { (range) (years) }\end{array}$ & $\begin{array}{l}62.0 \\
(18-85) \\
\end{array}$ & $\begin{array}{l}61.5 \\
(24-80) \\
\end{array}$ & $\begin{array}{l}63.0 \\
(18-85) \\
\end{array}$ \\
\hline \multirow[t]{2}{*}{ Gender } & Male & $44.0 \%$ & $40.0 \%$ & $46.7 \%$ \\
\hline & Female & $56.0 \%$ & $60.0 \%$ & $53.3 \%$ \\
\hline \multirow[t]{7}{*}{ Prior disease } & $\begin{array}{l}\text { Non-Hodgkin } \\
\text { lymphoma }\end{array}$ & $29.9 \%$ & $50.0 \%$ & $29.3 \%$ \\
\hline & Breast & $27.6 \%$ & $20.0 \%$ & $25.0 \%$ \\
\hline & Gastrointestinal & $6.0 \%$ & $5.0 \%$ & $7.6 \%$ \\
\hline & Hodgkin disease & $5.2 \%$ & $5.0 \%$ & $6.5 \%$ \\
\hline & Prostate & $5.2 \%$ & $0.0 \%$ & $6.5 \%$ \\
\hline & Testicular & $4.5 \%$ & $0.0 \%$ & $5.4 \%$ \\
\hline & Other & $21.6 \%$ & $20.0 \%$ & $19.6 \%$ \\
\hline \multirow{5}{*}{$\begin{array}{l}\text { Known } \\
\text { previous } \\
\text { treatment }\end{array}$} & Alkylator & $57.5 \%$ & $65.0 \%$ & $58.7 \%$ \\
\hline & $\begin{array}{l}\text { Topoisomerase } \\
\text { II inhibitor }\end{array}$ & $50.7 \%$ & $45.0 \%$ & $48.9 \%$ \\
\hline & Platinum & $20.9 \%$ & $20.0 \%$ & $21.7 \%$ \\
\hline & Radiation & $65.7 \%$ & $65.0 \%$ & $65.2 \%$ \\
\hline & Unknown & $8.2 \%$ & $15.0 \%$ & $5.4 \%$ \\
\hline Latency & $\begin{array}{l}\text { Median } \\
\text { (range) (years) }\end{array}$ & $\begin{array}{l}6.1 \\
(0.7-31)\end{array}$ & $\begin{array}{l}6.8 \\
(2.5-15.9)\end{array}$ & $\begin{array}{l}6.2 \\
(0.7-31)\end{array}$ \\
\hline \multirow[t]{2}{*}{ Diagnosis } & $\mathrm{AML}$ & $50.0 \%$ & $40.0 \%$ & $40.2 \%$ \\
\hline & MDS & $50.0 \%$ & $60.0 \%$ & $59.8 \%$ \\
\hline \multirow[t]{5}{*}{ Cytogenetics } & Deletion 5 & $29.9 \%$ & $55.0 \%$ & $30.4 \%$ \\
\hline & Deletion 7 & $33.6 \%$ & $70.0 \%$ & $32.6 \%$ \\
\hline & Complex & $41.0 \%$ & $70.0 \%$ & $39.1 \%$ \\
\hline & MLL abnormality & $10.4 \%$ & $5.0 \%$ & $8.7 \%$ \\
\hline & Other/unknown & $38.8 \%$ & $0.0 \%$ & $41.3 \%$ \\
\hline $\begin{array}{l}\% \text { blasts in } \\
\text { the bone } \\
\text { marrow }\end{array}$ & $\begin{array}{l}\text { Median (range) } \\
(\%)\end{array}$ & $14(0-95)$ & $8(1-80)$ & $9(0-91)$ \\
\hline \multirow{4}{*}{$\begin{array}{l}\text { Most } \\
\text { intensive } \\
\text { treatment } \\
\text { regimen }\end{array}$} & $\begin{array}{l}\text { Allogeneic } \\
\text { transplant }\end{array}$ & $37.3 \%$ & $30.0 \%$ & $41.3 \%$ \\
\hline & Myeloablative & $21.6 \%$ & $30.0 \%$ & $15.2 \%$ \\
\hline & $\begin{array}{l}\text { Non- } \\
\text { myeloablative }\end{array}$ & $30.6 \%$ & $30.0 \%$ & $34.8 \%$ \\
\hline & Other/unknown & $10.4 \%$ & $10.0 \%$ & $8.7 \%$ \\
\hline \multirow[t]{3}{*}{ Remission } & Yes & $49.3 \%$ & $30.0 \%$ & $53.3 \%$ \\
\hline & No & $48.5 \%$ & $70.0 \%$ & $45.7 \%$ \\
\hline & Unknown & $2.2 \%$ & $0.0 \%$ & $1.1 \%$ \\
\hline $\begin{array}{l}\text { Overall } \\
\text { survival }\end{array}$ & $\begin{array}{l}\text { Median } \\
\text { (range) (days) }\end{array}$ & $\begin{array}{l}385 \\
(6-3427)\end{array}$ & $\begin{array}{l}187.5 \\
(32-3427)\end{array}$ & $\begin{array}{l}424 \\
(6-2432)\end{array}$ \\
\hline
\end{tabular}

clones appears to be higher than clones with mutations in other DNA damage response genes, particularly PPM1D and SRCAP.

\section{Discussion}

In this study, we show that hematopoietic clones harboring mutations in DNA damage response genes expand in response to cytotoxic stress. The expansion of TP53 and PPM1D mutant clones has previously been observed after cytotoxic therapy in patients with either solid or hematologic malignancies ${ }^{7,8}$. Our data suggest that mutations in DNA damage response genes other than TP53 and PPM1D may also provide a fitness advantage to HSCs with cytotoxic therapy. In the earlier studies, mutations in some of these genes (e.g., ATM, BRCC3, etc.) were identified in a small percentage of patients ${ }^{7,8}$. Utilizing the sensitivity of errorcorrected sequencing, we show that clonal hematopoiesis involving such mutations is more frequent after cytotoxic therapy than previously observed. We also ascertain for the first time that mutations in SRCAP are frequently seen in patients following cytotoxic therapy. SRCAP is an ATP-dependent chromatin remodeler, which catalyzes the incorporation of variant H2A.Z histones into the nucleosome $e^{26,27}$. Interestingly, this protein has been shown to play a role in the repair of double-strand breaks resulting from genotoxic stress 9 . The 29 SRCAP variants identified in pheresis and/or post-transplant peripheral blood samples were scattered throughout the gene with $12(41.4 \%)$ truncating nonsense or frameshift mutations and $16(55.2 \%)$ missense mutations, suggesting they may be primarily loss-of-function. Of note, since we did not survey all DNA damage response genes, we may be underestimating the frequency of clonal hematopoiesis involving mutations in such genes following cytotoxic therapy. Indeed, Coombs et al. also identified mutations in the TP53 regulator CHEK2 in patients with solid malignancies ${ }^{7}$.

In most patients with clonal hematopoiesis following cytotoxic therapy, we identified multiple variants. This has been observed to a lesser degree in other studies of clonal hematopoiesis after cytotoxic therapy ${ }^{7,8}$ and in healthy individuals ${ }^{2-4}$. Determining whether these variants represent independent clones would require single-cell sequencing. However, the number of variants identified in individual patients (with many located in the same gene) and the wide distribution of their VAFs (Supplemental Data 2) suggest that at least some arise from independent clones. Consistent with this conclusion, Gibson et al. described patients with multiple variants after cytotoxic therapy who later developed $\mathrm{t}$-AML/t-MDS without all the variants present in the malignant clone $^{8}$. In patients with prior exposure to cytotoxic therapy, the presence of a mutation in a DNA damage response gene above the limit of detection was associated with a higher total number of identified variants. This observation raises the possibility that the expansion of hematopoietic clones harboring mutations in TP53, $P P M 1 D$, and other DNA damage response genes may serve as a biomarker of prior genotoxic stress. Several studies have shown that clonal hematopoiesis in patients following cytotoxic therapy is associated with a modestly increased risk of developing a therapy-related myeloid neoplasm ${ }^{8,22,23}$. Whether the presence and/or number of expanded clones with mutations in DNA damage response genes better defines the risk for therapy-related myeloid neoplasms will require further study.

In studies involving individuals with no known hematologic malignancy or with aplastic anemia, expanded hematopoietic clones have often been found to persist for years, often at stable VAFs, suggesting that they originated in an HSC or long-term progenitor ${ }^{3,6,16}$. Indeed, we show that mutant clones that have expanded following cytotoxic therapy typically exhibit multilineage potential, expanding initially through the myeloid lineages. These clones also were typically long-lived, persisting following autologous transplantation. In total, these data suggest that hematopoietic clones expanding under the stress of cytotoxic therapy typically arise from HSCs.

Our data suggests that different cellular stressors favor distinct hematopoietic clones harboring specific mutations and is consistent with murine models of how mutant HSCs respond to cellular stress. HSCs with loss of the DNA damage response gene $\operatorname{Trp53}$ gain a fitness advantage with genotoxic stress potentially through p53-mediated cell competition ${ }^{24,28}$. However, transplantation-induced stress does not provide them with a significant competitive advantage $e^{24,28-30}$. In contrast, Dnmt3a deficient HSCs gain a repopulating advantage over their wild-type counterparts with serial transplantation ${ }^{31}$. In humans, expanded DNMT3A mutant clones have been identified in aged individuals $^{2-4}$. They have also been observed actively expanding in the setting of aplastic anemia, where they portend a poor prognosis ${ }^{6}$. The cellular stressors influencing DNMT3A mutant clonal expansion in these two cases and how they relate to transplantation-induced stress remain unknown.

Our findings are also consistent with observations of patients undergoing hematopoietic transplantation. In patients receiving 
a

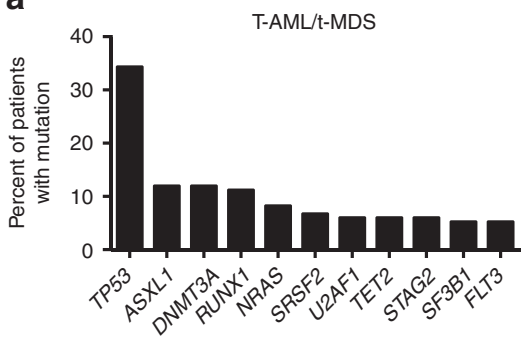

C

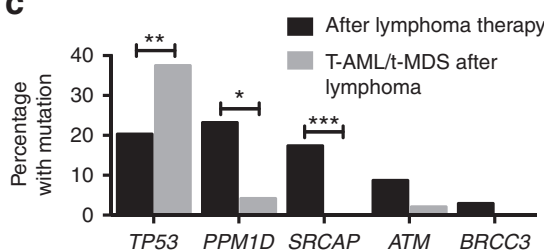

b

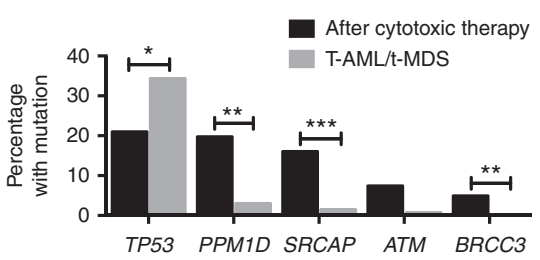

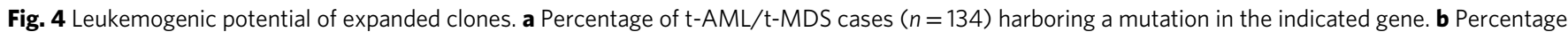
of patients who after cytotoxic therapy $(n=81)$ had an expanded clone harboring a variant in the indicated DNA damage response gene compared to the percentage of t-AML/t-MDS patients $(n=134)$ with a mutation in the same gene. $\mathbf{c}$ Same as $\mathbf{b}$ except that the comparison is between lymphoma patients at the time of autologous transplant $(n=69)$ and $t-A M L / t-M D S$ patients with a primary diagnosis of lymphoma $(n=48)$. For $\mathbf{b}$, $\mathbf{c}$ significance was determined with an exact logistic regression through use of a joint test. ${ }^{\star} P<0.05 ;{ }^{\star \star} P<0.01 ;{ }^{\star \star \star} P<0.001$

an autologous transplant, the risk of $\mathrm{t}-\mathrm{AML} / \mathrm{t}-\mathrm{MDS}$ is related more to the extent of pre-transplant cytoreductive therapy than transplantation itself ${ }^{32-34}$, potentially because transplantationinduced selection pressure does not provide a further fitness advantage to the mutant hematopoietic clones expanding with cytotoxic therapy. In the case of allogeneic transplantation, donor-derived DNMT3A mutant hematopoietic clones have been identified both in recipients with unexplained cytopenias after transplant and in recipients developing donor cell leukemia ${ }^{35-38}$. The role of screening potential allogeneic donors, particularly those of advanced age, for clonal hematopoiesis will be an area of future research interest, particularly given the transplantability of certain mutant clones.

Finally, our sequencing of t-AML/t-MDS suggests that the leukemogenic potential of expanded hematopoietic clones is strongly dependent on the specific mutations they harbor with TP53 mutant clones having a higher propensity towards leukemic evolution than clones with mutations in other DNA damage response genes, including PPM1D. In contrast to our findings, Lindsley et al. identified a high frequency (15\%) of PPM1D mutations in $t-\mathrm{MDS}^{39}$. A potential explanation for this discrepancy is the possibility that long-lived expanded non-leukemic clones may co-exist with leukemic clones (Supplementary Fig. 8). Supporting this is the observation that non-leukemic clones harboring mutations in TP53, DNMT3A, PPM1D, SRCAP and ZNF318 may rapidly expand with treatment of the AML/MDS clone $^{40-42}$. In the Lindsley et al. study, the median VAF of the largest PPM1D clone was only 5.5\% compared to $14.5 \%$ for TP53, with over half the patients analyzed after receiving treatment for their MDS $^{39}$. Thus, some PPM1D mutations may be unrelated to the founding MDS clone and instead represent co-existing nonleukemic clonal hematopoiesis. Despite being distinct from the leukemic clone, non-leukemic expanded clones may still inform on its evolution (e.g., suggest a previous exposure to genotoxic stress) and provide prognostic information.

In summary, our data show that cellular stressors result in the expansion of hematopoietic clones carrying specific mutations. Genotoxic stress from cytotoxic therapy promotes the expansion of clones with mutations in DNA damage response genes, such as TP53 and PPM1D. Transplantation-related stress may select for clones with DNMT3A mutations, particularly in codon 882 . In the future, longitudinal studies of patients before and after specific stressors (e.g., cytotoxic therapy, mobilization \pm cytotoxic stimulation, transplantation, etc.) will more precisely define their influence on clonal expansion and distinguish the impact of these stressors from that of other factors (e.g., pre-existing malignancies). Expanded clones are often long-lived and transplantable, but their leukemogenic potential varies, in part, due to the mutations they harbor. Most expanded clones, even those harboring TP53 mutations, do not evolve into leukemia. The contribution of other cellular stressors, such as inflammation, to the development of clonal hematopoiesis and the role that cellextrinsic stressors and/or cell-intrinsic genetic (or epigenetic) alterations play in the progression from clonal hematopoiesis to leukemia remain open and important questions.

\section{Methods}

Patient characteristics. Lymphoma patients undergoing autologous stem cell transplantation with available pheresis samples were consented under IRB protocol \#201108251. Lymphoma patients were analyzed based on sample availability. Myeloma patients undergoing an autologous stem cell transplant and allogeneic stem cell transplant donors with available pheresis samples were consented under IRB protocol \#201102270. Myeloma patients and healthy donors were chosen to allow for age and gender-matching with the lymphoma cohort. No data were excluded from the analysis. T-AML/t-MDS patients were selected from a larger cohort of adult AML and MDS patients enrolled in a single institution tissue banking protocol that was approved by the Washington University Human Studies Committee (IRB protocol \#201011766). Written informed consent for sequencing, including whole-genome sequencing, was obtained from all study participants. Patients were treated in accordance with National Comprehensive Cancer Network guidelines (http://www.nccn.org) with an emphasis on enrollment in therapeutic clinical trials whenever possible.

Patients were considered to have a previous history of cytotoxic therapy exposure if they previously received an alkylator, topoisomerase II inhibitor, or platinum-based chemotherapy or radiation therapy. Cytotoxic agents given as part of the pheresis mobilization regimen were not considered as a prior exposure to cytotoxic therapy given the short time period between this exposure and pheresis collection. Overall, 81 patients with analyzed pheresis samples had prior exposure to cytotoxic therapy, and 57 individuals lacked such exposure. To detect a change in the incidence of clonal hematopoiesis $\geq 0.1 \%$ VAF from $50 \%$ without cytotoxic therapy to $75 \%$ after cytotoxic therapy exposure with $80 \%$ power at 0.05 significance, we had calculated a desired sample size of 58 individuals per cohort. Clinical data for all patients in this study are included in Tables 1 and 2, Supplementary Table 1, and Supplementary Datas 1 and 4. 
Error-corrected HaloPlex sequencing. Error-corrected sequencing was performed using the HaloPlex HS Target Enrichment System (Agilent Technologies) as previously described ${ }^{43}$ with modifications as noted below. Briefly, as per Version $\mathrm{Cl}$ of the manufacturer's protocol, up to $500 \mathrm{ng}$ of genomic DNA was digested with a custom restriction enzyme cocktail in eight separate reactions and subsequently hybridized to a customized HaloPlex HS probe library $\times 2$ hours. This library was designed using the Agilent SureDesign platform to target 46 genes (Supplementary Table 2). The probes had dual indices: a unique molecular barcode to allow for error-corrected sequencing and a sample index to allow for sample multiplexing. Hybridized genomic DNA fragments were subsequently ligated to the library probes, captured with streptavidin, and amplified with PCR $(\times 24$ cycles $)$, creating read families, each with its own unique molecular barcode index. Library quality was assessed with the Agilent 2100 Bioanalyzer. Library concentration was assessed with qPCR according to the manufacturer's protocol (Kapa Biosystems). Libraries were normalized, pooled, and sequenced on the HiSeq 4000 with 15 samples sequenced per lane. On average, each sample had 40.7 million \pm 13.0 million mapped reads.

Molecularly barcoded reads were aligned with BWA MEM v0.7.9a, using parameters "-t 12, -M". SNVs were detected using BarCrawler, a custom GATK walker (https://github.com/abelhj/gatk/blob/master/public/external- example/src/ main/java/org/abelhj/WalkerTR1203.java), using parameters "-mmq 20 -mbq 20minCtBC 3 -dcov 500000 -discardN 1 -minOffset 5 -maxNM 3". Filters were applied to remove artifacts appearing at homopolymer runs of length greater than 4 and alignment artifacts appearing in greater than $5 \%$ of a panel of normal samples. Next, background noise calculation was performed on a position-byposition basis for each identified variant as follows. For each variant, read counts were gathered from all other samples, excluding those sites with a VAF above $25 \%$, which were assumed to be germline single nucleotide polymorphisms. The $\mathrm{R}$ statistical package was used to obtain a $p$-value via Fisher's exact test, comparing the reference and variant reads at a site to the number of reference and variant reads at that site in all other samples. Multiple testing correction was then applied with the $p$ adjust function (default parameters). Those variants with an adjusted $p$ value of less than 0.1 were retained. The same process was then repeated with subsequent background calculations excluding all variants retained in previous rounds until no new variants were identified. SNVs were retained if they were coding or splice site mutations, had 500 or more total reads, had 4 or more supporting reads, had a VAF greater than or equal to $0.1 \%$, and were not present in the Exome Aggregation Consortium database at a frequency greater than $0.1 \%$. Finally, three variants (CREBBP L2067, SETBP1 L284, and ZNF318 A1466S), which were present at varying frequencies in most samples tested, were excluded as artifacts.

Insertions and deletions were detected by using BarCrawler Consensus (https:// github.com/abelhj/gatk/blob/master/public/external-example/src/main/java/org/ abelhj/WalkerTRConsensus.java) to assemble each read family into a consensus sequence (params: -dcov 500000). Then VarScan v2.3.6 (params --min-coverage 3 --min-reads 3 --min-var-freq 0.001) was run on the resulting bam file to identify putative indels. As with SNVs, variant calls were restricted to coding regions of the genome. A per-site background error rate calculation was based on the total counts of any indel appearing at each position within each batch, thus accounting for artifacts like slippage at homopolymer runs. A Fisher's exact test was used to compare each variant call to the background rate; then $\mathrm{p}$-values were corrected for multiple testing using the Bonferroni method. Indels with adjusted $p<0.01$ were retained. This process was applied iteratively, as with the SNVs above. To account for highly variable sites, variants were also excluded if their VAF did not exceed five times the median absolute deviation of all VAFs at that location. Review of known problematic positions like ASXL1 G545 show that despite high levels of background "noise", obvious sequencing artifacts were removed. Both SNVs and indels were identified without a priori knowledge of whether the sample of interest was a pheresis or post-transplant peripheral blood sample or whether the pheresis sample was banked after a previous exposure to cytotoxic therapy.

Statistical analyses. In determining the relationship between hematopoietic clonal expansion and an individual's clinical features (Supplementary Table 3), the outcomes of interest included the identification of clonal hematopoiesis with a VAF $\geq 0.1 \%$, the total number of variants identified, and the presence of a variant in the genes DNMT3A, PPM1D, TP53, SRCAP, TET2, ZNF318, or ATM. The covariates included age and a history of radiation therapy, chemotherapy, or cytoreductive therapy (i.e., either radiation therapy or chemotherapy). The relationships between the binary outcomes and the covariates were analyzed with exact logistic regression. This was done in preference to ordinary logistic regression due to the small sample sizes available for some combinations of outcomes and covariates. The relationship between each outcome and each covariate, both alone and in combination with age, was examined. The relationships between the number of mutations and the covariates, both alone and in combination with age, were analyzed with a negativebinomial regression analysis. This technique is not dependent on equal variances between groups and is appropriate when the outcome is a count variable.

The question of whether mutations in the DNA damage response genes TP53, $P P M 1 D$, SRCAP, BRCC3, and ATM had a similar distribution after cytotoxic therapy versus at $\mathrm{t}-\mathrm{AML} / \mathrm{t}-\mathrm{MDS}$ was examined with an exact logistic regression through use of a joint test of the hypothesis that their parameters are equal to zero.
The analyses were done with the logistics procedure of SAS/Stat software, Version 9.3 for Windows.

Cell sorting. Cryopreserved cells were thawed as previously described ${ }^{44} .1 .5 \times 10^{7}$ cells were stained by standard protocols with the following antibodies: anti-CD3 e450 (eBioscience; clone OKT3; catalog\# 48-0037-42; 55:1 dilution), anti-CD14 APC (eBioscience; clone 61D3; catalog\# 17-0149-42; 20:1 dilution), anti-CD15 FITC (Fisher; clone HI98; catalog\# BDB555401; 4.6:1 dilution), anti-CD16 PE (Fisher; clone 3G8; catalog\# BDB555407; 11:1 dilution), and anti-CD19 APC (Fisher, clone HIB19; catalog\# BDB555415; 14:1 dilution). They were sorted using a modified Beckman Coulter MoFlo. Genomic DNA was isolated with the QIAmp DNA Mini Kit (Qiagen, Venlo, The Netherlands).

Droplet digital PCR. All primers and probes for ddPCR were designed by Bio-Rad per MIQE guidelines. ddPCR was performed as previously described ${ }^{45}$. Specifically, PCR was performed with $900 \mathrm{nM}$ forward and reverse primers, $250 \mathrm{nM}$ mutant and wild-type probes, and $<2 \mathrm{ng} / \mu \mathrm{l}$ genomic DNA with restriction enzyme digestion per Bio-Rad recommendations. PCR was performed with annealing/ extension temperatures of $53.5-56.0^{\circ} \mathrm{C}$ for 40 cycles. For droplet generation and analysis, we used the Bio-Rad QX200 ${ }^{\mathrm{TM}}$ Droplet Digital ${ }^{\mathrm{TM}}$ PCR System. Calculation of the mutant allele fraction was performed as previously described ${ }^{24}$. Specifically, we only identified mutant alleles when present in droplets also lacking the reference allele. We then used Poisson statistics to determine the number of droplets containing a single allele (either reference or mutant) and the number of droplets containing a single mutant allele. The VAF was determined as the percentage of the single allele droplets containing the mutant allele. Using "mutant only" droplets to determine the mutant allele fraction significantly reduces artifact caused by DNA degradation that has resulted in the chemical alteration of one of the two DNA strands (i.e., guanosine oxidation, cytosine deamination, etc.).

Targeted library preparation. Automated dual-indexed libraries were constructed with $250 \mathrm{ng}$ of genomic DNA utilizing the KAPA HTP library prep kit (KAPA Biosystems) on the SciClone NGS platform (Perkin Elmer) for 96 tumor/normal pairs. The samples were fragmented on the Covaris LE220 (Covaris) targeting 250 bp inserts. Forty-eight of the libraries were pooled pre-capture generating a $5 \mu \mathrm{g}$ library pool. Each library pool was hybridized with a custom set of xGen Lockdown Probes (IDT) targeting a set of recurrently mutated genes (RMG) found in AML. An additional ten genes commonly identified by our error-corrected assay as mutated after cytotoxic therapy were added at an equal molar ratio to the RMG probes, thus representing all regions with a similar quantity (Supplementary Table 4). The libraries were hybridized for 4 hours at $47^{\circ} \mathrm{C}$ followed by stringent washing. Enriched ssDNA library fragments were amplified with KAPA HiFi HotStart polymerase and $200 \mathrm{nM}$ primers prior to sequencing. The concentration of each captured library pool was determined through qPCR according to the manufacturer's protocol (KAPA Biosystems) to produce cluster counts appropriate for the Illumina HiSeq4000 platform. The capture pools were normalized and pooled prior to data generation. One lane of $2 \times 150 \mathrm{bp}$ sequence data generated $\sim 1$ $\mathrm{Gb}$ of data per sample. Approximately $300 \times$ mean depth of coverage was achieved, and $99.9 \%$ of targets were covered to a minimum of $20 \times$.

Exome library preparation. Automated dual-indexed libraries were constructed with 250 ng of genomic DNA utilizing the KAPA HTP library prep kit (KAPA Biosystems) on the SciClone NGS platform (Perkin Elmer) for 19 tumor/normal pairs. The samples were fragmented on the Covaris LE220 (Covaris) targeting 250 $\mathrm{bp}$ inserts. Ten libraries were pooled pre-capture generating a $5 \mu \mathrm{g}$ library pool Each library pool was hybridized with the SeqCap EZ Human Exome Kit v3.0 (Roche Nimblegen) that targets over 200,000 genes spanning $\sim 64 \mathrm{Mb}$ of the human genome. In addition to the exome space, a custom set of xGen Lockdown Probes (IDT) was spiked into the hybridization reaction targeting a set of RMG found in AML. The additional probes were added at an equal molar equivalent to the probe concentration of the exome probes, thus representing the RMG regions in a similar quantity as the exome regions. The libraries were hybridized for 72 hours at $47^{\circ} \mathrm{C}$ followed by stringent washing. Enriched ssDNA library fragments were amplified with KAPA HiFi HotStart polymerase and $200 \mathrm{nM}$ primers prior to sequencing. The concentration of each captured library pool was accurately determined through qPCR according to the manufacturer's protocol (KAPA Biosystems) to produce cluster counts appropriate for the Illumina HiSeq2000 platform. Two lanes of $2 \times 100 \mathrm{bp}$ sequence data generated $\sim 7 \mathrm{~Gb}$ of data per sample. Approximately $55 \times$ mean depth of coverage was achieved, and $83 \%$ of targets were covered to a minimum of $20 x$.

Somatic variant analysis. Sequence data was aligned to reference sequence build GRCh37-lite-build37 using bwa mem ${ }^{46}$ version 0.7.10 (params: -t 8::) and then merged and deduplicated using picard version 1.113 (https://broadinstitute.github. io/picard/). SNVs were detected using the union of four callers: 1) samtools ${ }^{47}$ version r982 (params: mpileup -BuDs) intersected with Somatic Sniper ${ }^{48}$ version 1.0.4 (params: -F vcf $-\mathrm{G}-\mathrm{L}-\mathrm{q} 1-\mathrm{Q} 15$ ) and processed through false-positive filter v1 (params: --bam-readcount- version 0.4 --bam-readcount-min-base-quality 15 --min-mapping-quality 40 --min-somatic-score 40$),$ 2) $\operatorname{VarScan}^{49}$ version 2.3 .6 
filtered by varscan-high-confidence filter version v1 and processed through falsepositive filter vl (params: --bam-readcount-version 0.4 --bam-readcount-minbase-quality 15), 3) Strelka ${ }^{50}$ version 1.0.11 (params: isSkipDepthFilters =1), and 4) mutect $^{51}$ v1.1.4 (params: number-of-chunks $=50$ ). Indels were detected using the union of 4 callers: 1) GATK ${ }^{52}$ somatic-indel version 53362 2) pindel ${ }^{53}$ version 0.5 filtered with pindel somatic calls and VAF filters (params: --variant-freq-cutoff $=$ 0.08 ), and pindel read support, 3) VarScan ${ }^{49}$ version 2.3.6 filtered by varscan-highconfidence-indel version v1 and 4) Strelka ${ }^{50}$ version 1.0.11 (params: isSkipDepthFilters $=1$ ). SNVs and indels were further filtered by removing artifacts found in a panel of 905 normal exomes, removing sites that exceeded $0.1 \%$ frequency in the 1000 genomes or NHLBI exome sequencing projects, and then using a Bayesian classifier (https://github.com/genome/genome/blob/master/lib/perl/ Genome/Model/Tools/Validation/IdentifyOutliers.pm), retaining variants classified as somatic with a binomial log-likelihood of at least 10 . Variants present at low VAF in the 46 genes interrogated by our HaloPlex error-corrected sequencing assay were manually reviewed if they had a binomial log-likelihood of at least three and retained if they passed validation.

Data availability. Exome sequencing data of t-AML/t-MDS has been submitted to dbGAP (accession number phs000159.v9). All other relevant data are included in the article or supplementary files, or available from the authors upon request. Code used for both error-corrected and non-error-corrected variant identification are described in their applicable methodology sections with download sites listed where applicable.

Received: 28 September 2017 Accepted: 4 January 2018 Published online: 31 January 2018

\section{References}

1. Welch, J. S. et al. The origin and evolution of mutations in acute myeloid leukemia. Cell 150, 264-278 (2012).

2. Genovese, G. et al. Clonal hematopoiesis and blood-cancer risk inferred from blood DNA sequence. N. Engl. J. Med. 371, 2477-2487 (2014).

3. Jaiswal, S. et al. Age-related clonal hematopoiesis associated with adverse outcomes. N. Engl. J. Med. 371, 2488-2498 (2014).

4. Xie, M. et al. Age-related mutations associated with clonal hematopoietic expansion and malignancies. Nat. Med. 20, 1472-1478 (2014).

5. Steensma, D. P. et al. Clonal hematopoiesis of indeterminate potential and its distinction from myelodysplastic syndromes. Blood 126, 9-16 (2015).

6. Yoshizato, T. et al. Somatic mutations and clonal hematopoiesis in aplastic anemia. N. Engl. J. Med. 373, 35-47 (2015).

7. Coombs, C. C. et al. Therapy-related clonal hematopoiesis in patients with non-hematologic cancers is common and associated with adverse clinical outcomes. Cell Stem Cell 21, 374-382.e4 (2017).

8. Gibson, C. J. et al. Clonal hematopoiesis associated with adverse outcomes after autologous stem-cell transplantation for lymphoma. J. Clin. Oncol. 35 , 1598-1605 (2017).

9. Dong, S. et al. The human SRCAP chromatin remodeling complex promotes DNA-end resection. Curr. Biol. 24, 2097-2110 (2014).

10. Lu, X., Nannenga, B. \& Donehower, L. A. PPM1D dephosphorylates Chk1 and p53 and abrogates cell cycle checkpoints. Genes Dev. 19, 1162-1174 (2005).

11. Ciccia, A. \& Elledge, S. J. The DNA damage response: making it safe to play with knives. Mol. Cell 40, 179-204 (2010).

12. Birkenbihl, R. P. \& Subramani, S. Cloning and characterization of $\operatorname{rad} 21$ an essential gene of Schizosaccharomyces pombe involved in DNA doublestrand-break repair. Nucleic Acids Res. 20, 6605-6611 (1992).

13. Lal, R. et al. Somatic TP53 mutations characterize preleukemic stem cells in acute myeloid leukemia. Blood 129, 2587-2591 (2017).

14. Shlush, L. I. et al. Identification of pre-leukaemic haematopoietic stem cells in acute leukaemia. Nature 506, 328-333 (2014).

15. Corces-Zimmerman, M. R., Hong, W. J., Weissman, I. L., Medeiros, B. C. \& Majeti, R. Preleukemic mutations in human acute myeloid leukemia affect epigenetic regulators and persist in remission. Proc. Natl. Acad. Sci. USA 111, 2548-2553 (2014).

16. Young, A. L., Challen, G. A., Birmann, B. M. \& Druley, T. E. Clonal haematopoiesis harbouring AML-associated mutations is ubiquitous in healthy adults. Nat. Commun. 7, 12484 (2016).

17. Notaro, R., Cimmino, A., Tabarini, D., Rotoli, B. \& Luzzatto, L. In vivo telomere dynamics of human hematopoietic stem cells. Proc. Natl. Acad. Sci. USA 94, 13782-13785 (1997).

18. Lee, J. et al. Telomere length changes in patients undergoing hematopoietic stem cell transplantation. Bone Marrow Transplant. 24, 411-415 (1999).

19. Banfi, A., Bianchi, G., Galotto, M., Cancedda, R. \& Quarto, R. Bone marrow stromal damage after chemo/radiotherapy: occurrence, consequences and possibilities of treatment. Leuk. Lymphoma 42, 863-870 (2001).
20. Shirota, T. \& Tavassoli, M. Cyclophosphamide-induced alterations of bone marrow endothelium: implications in homing of marrow cells after transplantation. Exp. Hematol. 19, 369-373 (1991).

21. Flynn, C. M. \& Kaufman, D. S. Donor cell leukemia: insight into cancer stem cells and the stem cell niche. Blood 109, 2688-2692 (2007).

22. Gillis, N. K. et al. Clonal haemopoiesis and therapy-related myeloid malignancies in elderly patients: a proof-of-concept, case-control study. Lancet Oncol. 18, 112-121 (2017).

23. Takahashi, K. et al. Preleukaemic clonal haemopoiesis and risk of therapyrelated myeloid neoplasms: a case-control study. Lancet Oncol. 18, 100-111 (2017).

24. Wong, T. N. et al. Role of TP53 mutations in the origin and evolution of therapy-related acute myeloid leukaemia. Nature 518, 552-555 (2015).

25. Lindsley, R. C. et al. Acute myeloid leukemia ontogeny is defined by distinct somatic mutations. Blood 125, 1367-1376 (2015).

26. Watanabe, S. \& Peterson, C. L. The INO80 family of chromatin-remodeling enzymes: regulators of histone variant dynamics. Cold Spring Harb. Symp. Quant. Biol. 75, 35-42 (2010).

27. Wong, M. M., Cox, L. K. \& Chrivia, J. C. The chromatin remodeling protein, SRCAP, is critical for deposition of the histone variant H2A.Z at promoters. J. Biol. Chem. 282, 26132-26139 (2007).

28. Bondar, T. \& Medzhitov, R. p53-mediated hematopoietic stem and progenitor cell competition. Cell Stem Cell 6, 309-322 (2010).

29. Chen, J. et al. Enrichment of hematopoietic stem cells with SLAM and LSK markers for the detection of hematopoietic stem cell function in normal and Trp53 null mice. Exp. Hematol. 36, 1236-1243 (2008).

30. Liu, Y. et al. p53 regulates hematopoietic stem cell quiescence. Cell Stem Cell $\mathbf{4}$, 37-48 (2009).

31. Challen, G. A. et al. Dnmt3a is essential for hematopoietic stem cell differentiation. Nat. Genet. 44, 23-31 (2011).

32. Forrest, D. L. et al. High-dose therapy and autologous hematopoietic stem-cell transplantation does not increase the risk of second neoplasms for patients with Hodgkin's lymphoma: a comparison of conventional therapy alone versus conventional therapy followed by autologous hematopoietic stem-cell transplantation. J. Clin. Oncol. 23, 7994-8002 (2005).

33. Govindarajan, R. et al. Preceding standard therapy is the likely cause of MDS after autotransplants for multiple myeloma. Br. J. Haematol. 95, 349-353 (1996).

34. Hake, C. R., Graubert, T. A. \& Fenske, T. S. Does autologous transplantation directly increase the risk of secondary leukemia in lymphoma patients? Bone Marrow Transplant. 39, 59-70 (2007).

35. Gondek, L. P. et al. Donor cell leukemia arising from clonal hematopoiesis after bone marrow transplantation. Leukemia 30, 1916-1920 (2016)

36. Herold, S. et al. Donor cell leukemia: evidence for multiple preleukemic clones and parallel long term clonal evolution in donor and recipient. Leukemia 31, $1637-1640$ (2017).

37. Yasuda, T. et al. Leukemic evolution of donor-derived cells harboring IDH2 and DNMT3A mutations after allogeneic stem cell transplantation. Leukemio 28, 426-428 (2014)

38. Gibson, C. J. et al. Donor-engrafted CHIP is common among stem cell transplant recipients with unexplained cytopenias. Blood 130, 91-94 (2017).

39. Lindsley, R. C. et al. Prognostic Mutations in Myelodysplastic Syndrome after Stem-Cell Transplantation. N. Engl. J. Med. 376, 536-547 (2017).

40. da Silva-Coelho, P. et al. Clonal evolution in myelodysplastic syndromes. Nat Commun. 8, 15099 (2017).

41. Welch, J. S. et al. TP53 and decitabine in acute myeloid leukemia and myelodysplastic syndromes. N. Engl. J. Med. 375, 2023-2036 (2016).

42. Wong, T. N. et al. Rapid expansion of preexisting nonleukemic hematopoietic clones frequently follows induction therapy for de novo AML. Blood 127, 893-897 (2016).

43. Uy, G. L. et al. Dynamic changes in the clonal structure of MDS and AML in response to epigenetic therapy. Leukemia 31, 872-881 (2017).

44. Klco, J. M. et al. Functional heterogeneity of genetically defined subclones in acute myeloid leukemia. Cancer Cell. 25, 379-392 (2014).

45. Hindson, B. J. et al. High-throughput droplet digital PCR system for absolute quantitation of DNA copy number. Anal. Chem. 83, 8604-8610 (2011).

46. Li, H. \& Durbin, R. Fast and accurate short read alignment with BurrowsWheeler transform. Bioinformatics 25, 1754-1760 (2009).

47. Li, H. et al. The sequence alignment/Map format and SAMtools. Bioinformatics 25, 2078-2079 (2009).

48. Larson, D. E. et al. SomaticSniper: identification of somatic point mutations in whole genome sequencing data. Bioinformatics 28, 311-317 (2012).

49. Koboldt, D. C. et al. VarScan 2: somatic mutation and copy number alteration discovery in cancer by exome sequencing. Genome Res. 22, 568-576 (2012).

50. Saunders, C. T. et al. Strelka: accurate somatic small-variant calling from sequenced tumor-normal sample pairs. Bioinformatics 28, 1811-1817 (2012).

51. Cibulskis, K. et al. Sensitive detection of somatic point mutations in impure and heterogeneous cancer samples. Nat. Biotechnol. 31, 213-219 (2013). 
52. McKenna, A. et al. The Genome Analysis Toolkit: a MapReduce framework for analyzing next-generation DNA sequencing data. Genome Res. 20, 1297-1303 (2010).

53. Ye, K., Schulz, M. H., Long, Q., Apweiler, R. \& Ning, Z. Pindel: a pattern growth approach to detect break points of large deletions and medium sized insertions from paired-end short reads. Bioinformatics 25, 2865-2871 (2009).

\section{Acknowledgements}

This work was supported by National Institutes of Health, National Cancer Institute grants PO1 CA101937, (D.C.L. and T.J.L.), P50 CA171963 (D.C.L.) and K08 CA197369 (T.N.W.). Technical support was provided by the Alvin J. Siteman Cancer Center Tissue Procurement Core and the High-Speed Cell Sorting Core at Washington University School of Medicine, which are supported by National Cancer Institute grant P30 CA91842.

\section{Author contributions}

T.N.W. designed and performed the research, analyzed the data, and wrote the manuscript. C.A.M., M.R.M.J., A.P.S., E.J.D. and R.S.F. contributed to the generation and analysis of the HaloPlex error-corrected, exome, and targeted sequencing data. N.B., A.F. C., N.M.H., M.F., S.E.H., M.J., K.L., P.W. and R.V. collected and processed clinical data and tissue samples. J.D.B. performed statistical analyses of the clinical data. J.F.D., J.S.W., T.A.G., M.J.W. and T.J.L. contributed to data analysis. D.C.L. supervised all the research and edited the manuscript, which was approved by all co-authors.

\section{Additional information}

Supplementary Information accompanies this paper at https://doi.org/10.1038/s41467018-02858-0.
Competing interests: The authors declare no competing financial interests.

Reprints and permission information is available online at http://npg.nature.com/ reprintsandpermissions/

Publisher's note: Springer Nature remains neutral with regard to jurisdictional claims in published maps and institutional affiliations.

\begin{abstract}
(c) (i) Open Access This article is licensed under a Creative Commons Attribution 4.0 International License, which permits use, sharing, adaptation, distribution and reproduction in any medium or format, as long as you give appropriate credit to the original author(s) and the source, provide a link to the Creative Commons license, and indicate if changes were made. The images or other third party material in this article are included in the article's Creative Commons license, unless indicated otherwise in a credit line to the material. If material is not included in the article's Creative Commons license and your intended use is not permitted by statutory regulation or exceeds the permitted use, you will need to obtain permission directly from the copyright holder. To view a copy of this license, visit http://creativecommons.org/ licenses/by/4.0/.
\end{abstract}

(C) The Author(s) 2018

Terrence N. Wong ${ }^{1}$, Christopher A. Miller ${ }^{1,2}$, Matthew R.M. Jotte', Nusayba Bagegni', Jack D. Baty ${ }^{3}$, Amy P. Schmidt', Amanda F. Cashen ${ }^{1,4}$, Eric J. Duncavage ${ }^{5}$, Nichole M. Helton', Mark Fiala', Robert S. Fulton ${ }^{2}$, Sharon E. Heath', Megan Janke', Kierstin Luber', Peter Westervelt ${ }^{1,4}$, Ravi $\mathrm{Vij}^{1,4}$, John F. DiPersio, ${ }^{1,4}$ John S. Welch ${ }^{1,4}$, Timothy A. Graubert ${ }^{6}$, Matthew J. Walter ${ }^{1,4}$, Timothy J. Ley ${ }^{1,4} \&$ Daniel C. Link ${ }^{1,4}$

${ }^{1}$ Division of Oncology, Washington University School of Medicine, St. Louis, MO 63110, USA. ${ }^{2}$ McDonnell Genome Institute, Washington University School of Medicine, St. Louis, MO 63110, USA. ${ }^{3}$ Division of Biostatistics, Washington University, St. Louis, MO 63110, USA. ${ }^{4}$ Siteman Cancer Center, Washington University, St. Louis, MO 63110, USA. ${ }^{5}$ Department of Pathology and Immunology, Washington University School of Medicine, St Louis, MO 63110, USA. ${ }^{6}$ Massachusetts General Hospital, Boston, MA 02114, USA 